\title{
Retrieval of information from multiple ensembles in short-term memory*
}

\author{
RONALD D. CRAIN and DONALD V. DeROSA $\dagger$ \\ Bowling Green State University, Bowling Green, Ohio 43403
}

\begin{abstract}
A recognition memory experiment investigated Ss' ability to organize information in short-term memory. A paradigm similar to that used by Sternberg was employed. A sequentially presented series of six digits (positive set) was shown with each digit appearing on a red, green, or amber background. The colors defined different ensembles, and responding to a test digit was contingent upon an item's membership in the positive set and the color-defined ensemble. Reaction time (RT) to the test digits indicated that Ss did organize information into ensembles. Furthermore, when informative cues were presented prior to the test item, Ss directed and confined their search to the cued subset.
\end{abstract}

Sternberg (1966) introduced a method for investigating the search strategies used by $S s$ in a recognition memory task. Ss were shown a sequentially presented set of homogeneous stimuli (digits), called the positive set, and then shown a test or probe stimulus to which they were instructed to make a first response (positive) if the probe stimulus was identical to any member of the positive set and a second response (negative) otherwise. It was found that reaction time (RT) increased linearly as a function of the size of the positive set for both positive and negative responses, with the slopes of both functions being equal. These findings led Sternberg to propose a theory of high-speed scanning in which an internal representation of the test item is compared successively to the items in memory at an average rate of approximately 25 to 30 items per second. A positive response is made if there has been a match, and a negative response is made otherwise. Since $\mathrm{RT}$ increased with positive set size at the same rate for positive and negative responses, the search was postulated to be exhaustive rather than self-terminating. If the search were terminated when a match was made, the positive probes should have resulted, on the average, in half as many comparisons as the negative probes, reducing the slope by one-half. Sternberg (1969) reported equivalent results for both long-term memory (LTM) and short-term memory (STM), and hypothesized that "the same memory system was being scanned: that is, when information in inactive memory ... [LTM] has to be used, it may be entered also in active memory ... ind thus becomes more readily available [p. 430]."

Recently, variations of Sternberg's paradigm have seen used to study the organization of information in 3TM and LTM. In an LTM study, Forrin and Morin 1967) demonstrated that stimuli in sets composed of

\footnotetext{
*This investigation was supported by National Science oundation Grant GB-16729 awarded to Bowling Green State Iniversity and the second author.

$\dagger$ Requests for reprints should be sent to Donald V. DeRosa, Jepartment of Psychology, Bowling Green State University, 3owling Green, Ohio 43403.
}

letters and digits could be named faster if Ss were informed of an item's set (number or symbol) membership prior to presentation. Briggs and Swanson (1969) also employed a naming task using sets of random figures with $2,4,8$, or 16 items in a subset. All figures within a subset had the same number of sides. Each figure was assigned a name consisting of a letter and a two-digit number, such as A-22. The names of all figures within a subset started with the same letter. They found that RT increased with subset size. Furthermore, when Ss were informed of the subset from which the test item would be drawn (cued naming), RTs were faster than those observed on noncued trials. The reduction in RT resulting from informative cues did not interact with subset size, indicating that subset selection and scanning within the subset are independent processes. These findings are consistent with Oldfield's (1966) hypothesis that information in LTM is organized into subsets (e.g., frequently used words vs less frequently used words) and that memory search involves two steps: first, the selection of the appropriate subset to be searched and then a search within the subset itself.

Williams (1971) varied both the number of subsets or "ensembles" in memory and the memory load within ensembles. Each of three groups of Ss learned three different ensembles which were differentiated by background colors. For the three groups, the ensembles contained two, three, and four letters, respectively. The size of the total memory set was controlled by telling the $\mathrm{S}$ which ensembles were to be used. Test stimuli were presented, and the $S$ was to indicate whether the test stimulus was a member of the probed ensemble. Reaction time increased with the memory load within ensembles and with the number of ensembles in memory. There was no interaction between the number of ensembles in memory and memory load within ensembles, again indicating that search is a two-stage process.

Sternberg's method has also been used to study similar types of organization in STM. Milles (1969) presented positive sets of one, two, three, or four items 
consisting of all digits, all letters, or a combination of digits and letters. When the positive set contained both letters and digits, the slope of the function relating RT to positive set size was less than the slope for the comparable function for positive sets containing homogeneous items. These data indicate that categorization within the positive set facilitated retrieval. Naus, Glucksberg, and Ornstein (1972) obtained similar results, and demonstrated that the decrease in slope was the result of a reduction in the number of comparisons made in the two-category list. Kaminsky and DeRosa (1972) presented sets of six items, with two, three, or four of the items being digits and the remainder letters. Reaction time increased as a function of probed subset size, indicating a directed search strategy which was confined to the probed subset. Reaction time was also found to be faster when an informative cue was presented than when a noninformative cue was shown.

The results of these studies indicate that $S$ are capable of organizing information in memory into subsets for both LTM and STM, and employ a directed, two-stage search strategy through these subsets when attempting to retrieve information from memory. However, such organization has been shown in STM only for subsets that have been highly overlearned (e.g., digits and letters), and are differentiated by the items within them rather than by an independent attribute. It is possible that these items are stored as separate subsets in LTM. Both Posner (1967) and Sternberg (1969) have suggested that information in LTM can be transferred to STM. It could be possible that in these studies of organization in STM, memory representations of the items shown in the positive set were transferred from LTM to STM, retaining the same organizational pattern (i.e., as two separate subsets). The purpose of the present study was to determine whether Ss can actively organize information in STM into subsets that have not been overlearned, are not present as subsets in LTM, and for which the basis of subset formation is independent of the items themselves.

\section{METHOD}

\section{Subjects}

The Ss were 10 undergraduates, 3 males and 7 females, who were paid for their participation. Each $\mathrm{S}$ served in all conditions of the experiment.

\section{Stimuli and Apparatus}

Each $S$ was tested individually in a sound-deadened chamber. The stimuli were presented visually on a single display cell (IEE Series 865 ) mounted on a display panel approximately $2 \mathrm{ft}$ from $\mathrm{S}$. The stimuli were single digits $.50 \mathrm{in}$. in height, selected from the set of digits $1-9$, with a background color surrounding each digit. The background colors were selected from three hues-red, green, and amber. Ss responded verbally, and responses were detected by a voice-operated relay. Accuracy feedback was provided by means of two lights mounted on opposite sides of the display. Reaction time was measured by a Hunter Klockounter to the nearest $.001 \mathrm{sec}$.

\section{Experimental Conditions and Procedure}

On each experimental trial, the Ss were shown a sequentially presented set of six stimuli which defined the positive set. Each stimulus in the positive set was presented for $0.7 \mathrm{sec}$, with a 0.7 -sec interval between successive stimuli. Seven-tenths of a second after the last stimulus had been presented, a cue was displayed and remained on for $0.7 \mathrm{sec}$. Two seconds after termination of the cue, the probe stimulus appeared and remained on until $S$ responded. The $S$ was instructed to say "yes" if the probe was a member of the positive set and "no" otherwise. In order for S to respond "yes" to a test probe, the digit must have appeared with the same background color in the positive set. Two types of stimuli required a "no" response: (a) stimuli for which the digit did not appear with that background color or any other background color in the positive set, i.e., the digit was not a member of any ensemble in memory (called an N1 response), and (b) stimuli for which the digit appeared with a different background color in the positive set, i.e., the digit was a member of an ensemble in memory defined by a different background color (called an N2 response). The test probes always contained the background color of one of the ensembles in memory. After $S$ had responded, the appropriate feedback light was shown.

Four variables were manipulated: (a) the number of ensembles in memory (defined by background colors), (b) ensemble size, (c) type of cue (informative vs noninformative), and (d) type of presentation (grouped vs nongrouped arrangement of ensemble members). The positive set consisted of either two or three ensembles. When the positive set contained two ensembles, ensemble size ranged from one to five. When the positive set consisted of three ensembles, ensemble size ranged from one to four. For example, a positive set consisting of the stimuli 2 (amber), 4 (red), 6 (amber), 8 (amber), 1 (red), 3 (green) would contain three ensembles (amber, red, and green) with three, two, and one item in each ensemble, respectively. The two-ensembles and three-ensembles conditions each had three different combinations of ensemble sizes. When there were two ensembles in memory, the ensemble sizes were one and five items, two and four items, or three and three items. With three ensembles in memory, ensemble sizes were one, one, and four items, one, two, and three items, or two, two, and two items.

For one-half of the trials there was an informative cue preceding the test item. The informative cue was the presentation of the color of the background of the probe which would follow. For the remaining trials, a noninformative cue preceded the probe. The noninformative cue was the presentation of a white field, indicating only that the probe would follow. On one-half of the trials the items in the positive set were presented in a grouped order. That is, all the items from a particular ensemble were presented contiguously. On the remaining trials a nongrouped arrangement of items was used.

All conditions were counterbalanced as completely as possible. A total of 864 different trials was constructed. For each trial, the digits and ensemble colors associated with the digits were chosen randomly. The grouped trials and the nongrouped trials were separately randomized and then divided into 24 blocks (12 grouped and 12 nongrouped) of 36 trials each. For each S, a randomly determined order of grouped blocks was alternated with a randomly determined order of nongrouped blocks. Each $S$ responded to each block of trials twice (after all 24 blocks had been shown, the sequence was repeated), resulting in 1,728 experimental trials per $S$. Over trials, the probability that the test item was a positive item was 5 .

There was also a baseline condition, in which all items in the positive set were members of the same ensemble (all items had the same background color), but with the positive set consisting of one, two, three, four, or five items. There were 240 different baseline trials. For each trial, the digits and the background color were chosen randomly. These trials were randomized and divided 
into six blocks of 40 trials each. Again, the order of presentation of the blocks was determined randomly for each S. Each S responded to each block of trials once. The baseline trials were administered after all the experimental trials had been completed.

Ss were tested in seven $1 \frac{1}{2}-\mathrm{h}$ sessions. Each $\mathrm{S}$ received preliminary training (approximately $1 \mathrm{~h}$ ) on the task. Since only correct responses were used in the data analyses, only those Ss whose error rate in the training phase was below $20 \%$ were used in the study.

\section{RESULTS AND DISCUSSION}

Mean RTs for correct responses were computed separately for each $\mathrm{S}$. The average error rate over Ss was $4.7 \%$. The error rate across Ss ranged from $1.1 \%$ to $7.4 \%$. The data were analyzed in an attempt to determine if Ss can organize new information in STM and utilize that organization in retrieval. In addition, interest focused on the factors that influence the organization and retrieval process.

\section{One Ensemble in Memory}

In keeping with Sternberg's (1966) model, it was expected that Ss would use a serial exhaustive search strategy when there was only one ensemble in memory. Figure 1 shows RT plotted as a function of ensemble size for the baseline condition. Reaction time increases significantly with ensemble size, $F(4,36)=106.17$, $p<.001$. Both the linear and cubic components of the ensemble size variable are significant, $F(1,9)=184.16$, $p<.001$ and $F(1,9)=11.89, p<.01$, respectively. The slope of the best fitting straight line (found by the least-squares solution) for the average curve (positive and negative responses combined) is $34 \mathrm{msec}$ per unit increase in set size. The best fitting straight line accounts for $98.5 \%$ of the variance. The slope constant presumably reflects the time taken by the comparison component of the retrieval process. These data therefore indicate that the Ss searched through the positive set at a rate of approximately 29 items per second, which agrees closely with the rates found by Sternberg $(1966,1969)$ and Kaminsky and DeRosa (1972). The negative responses are significantly slower than the positive responses, $F(1,9)=14.91, p<.005$. The slope of the best fitting straight line for the negative response condition is $32 \mathrm{msec}$ per unit increase in set size, while the slope for the positive response condition is $35 \mathrm{msec}$ per unit increase in set size. The interaction of Type of Response by Ensemble Size is not significant, $F(4,36)=$ $1.73, \mathrm{p}>.05$. The slopes are therefore essentially identical, indicating that the hypothesis that the two functions are parallel must be retained. These findings support the notion that the search was serial and exhaustive when there was one ensemble in memory.

\section{Multiple Ensembles in Memory}

If Ss organized the to-be-remembered information

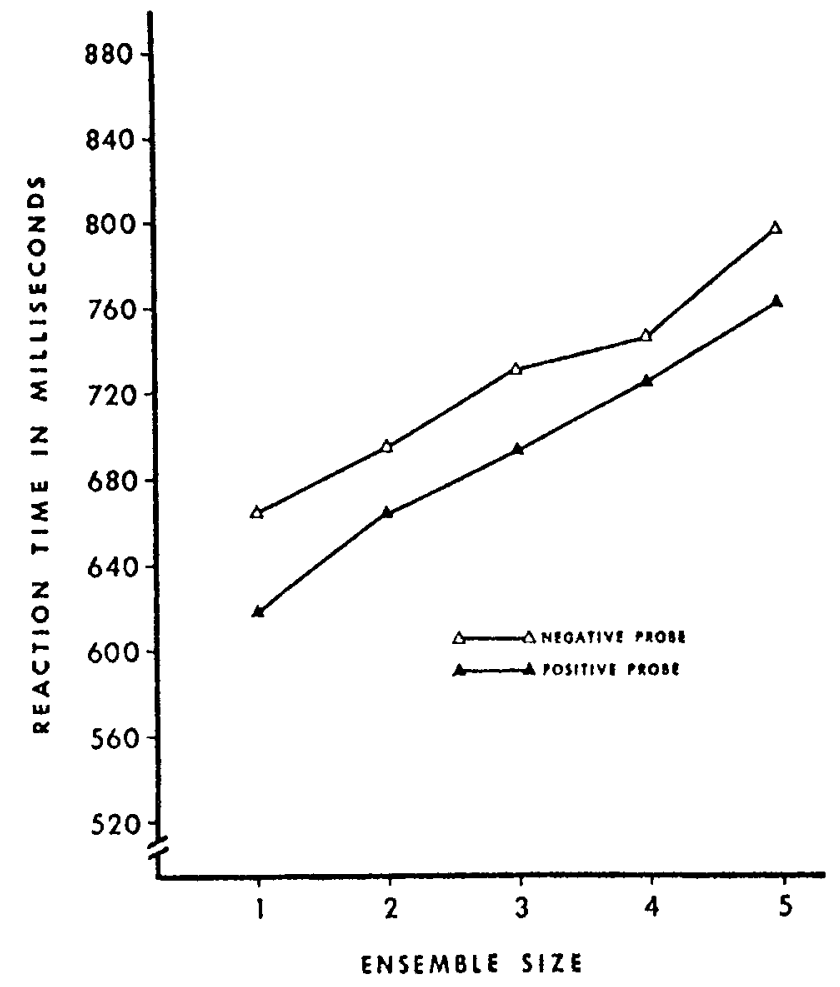

Fig. 1. Mean RT as a function of ensemble size for positive and negative probes in the baseline condition.

into subsets in memory, they might have directed their search to only the probed ensemble. If so, RT should increase with increases in probed ensemble size. However, if the Ss searched not only through the probed ensemble, but through the entire positive set, then RT should not increase with increases in probed ensemble size, since six items would be searched each time. In Fig. 2, RT is plotted as a function of probed ensemble size for the experimental and baseline conditions. For the experimental condition RT does increase with probed ensemble size, $F(4,36)=26.91, p<.001$. Both the linear and quadratic components are significant, $F(1,9)=30.31, p<.001$ and $F(1,9)=17.34, p<.005$, respectively. These data provide evidence that the Ss did organize the information and searched through the appropriate ensemble. However, the slope of the function relating RT to probed ensemble size for the experimental conditions is $23 \mathrm{msec}$ per unit increase in ensemble size, compared to $34 \mathrm{msec}$ per unit increase in ensemble size for the baseline condition. This could result from the $\mathrm{Ss}^{\prime}$ not searching only through the probed ensemble when there is more than one ensemble in memory, or from the use of different search strategies for some of the experimental conditions (e.g., self-terminating rather than exhaustive search).

As a preliminary examination of these two possibilities, the data for the experimental conditions were replotted in Fig. 3. The left panel of Fig. 3 shows RT as a function of probed ensemble size for positive and negative responses separately. The negative 


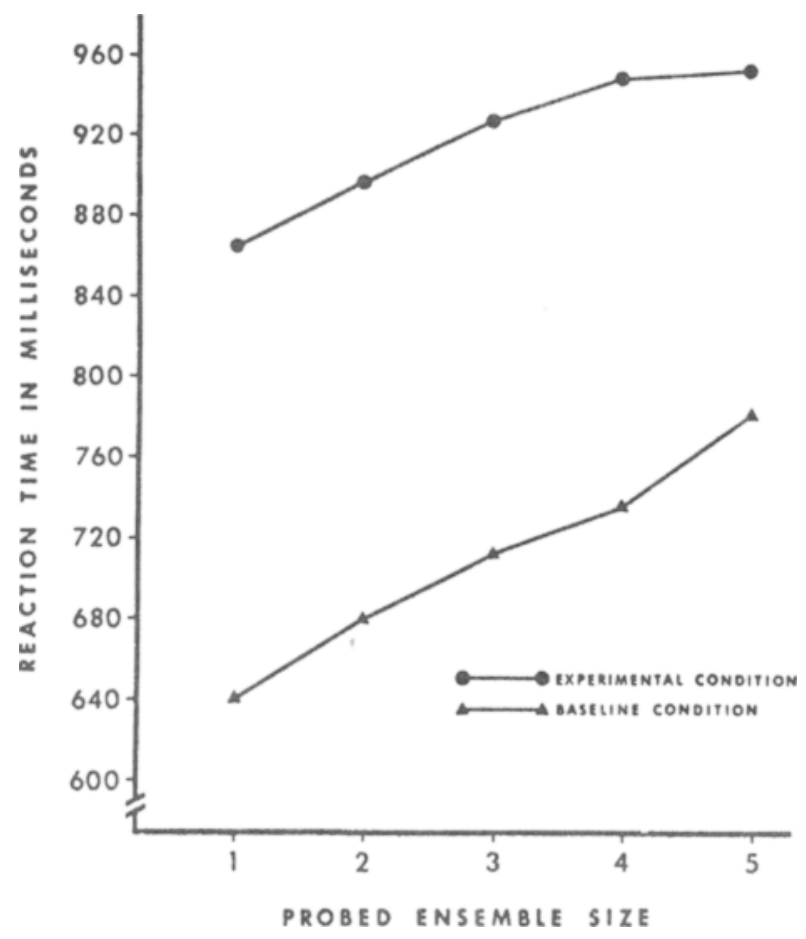

Fig. 2. Mean RT as a function of ensemble size in the baseline condition and probed ensemble size in the experimental condition.

responses are again significantly slower than the positive responses, $F(1,9)=46.11, p<.001$. The slopes of the best fitting straight lines for the negative response condition and the positive response condition are $20 \mathrm{msec}$ and $26 \mathrm{msec}$ per unit increase in ensemble size, respectively. The Response Type by Probed Ensemble Size interaction is significant, $F(4,36)=4.06, p<.01$. However, the linear component of this interaction is not significant, $F(1,9)=4.96, p>.05$. Therefore, the hypothesis that the functions are parallel can be retained. The right panel of Fig. 3 shows RT plotted as a function of probed ensemble size for the N1 and N2 responses separately. If the Ss searched only through the probed ensemble, and were unaffected by the items within the other ensembles, then the function relating $\mathrm{RT}$ to probed ensemble size for the $\mathrm{N} 1$ responses (the digit was not seen in any ensemble) should be the same as the function relating RT to probed ensemble size for the $\mathrm{N} 2$ responses (the digit was seen in a different ensemble). The $\mathrm{N} 2$ responses were significantly slower than the N1 responses, $\mathrm{F}(1,9)=25.50, \mathrm{p}<.001$. The overall mean RT for the N1 responses is $934 \mathrm{msec}$, while the comparable figure for the $\mathrm{N} 2$ responses is $972 \mathrm{msec}$, a difference of $38 \mathrm{msec}$. There is also a significant Response Type by Probed Ensemble Size interaction, $\mathrm{F}(4,36)=11.24, \mathrm{p}<.001$. It can be seen in Fig. 3 that when the probed ensemble size was five, the RT for the $\mathrm{N} 2$ condition dropped and was faster than the RT for the $\mathrm{N} 1$ condition. When the probed ensemble size was five, there were two ensembles in memory (never three), and the test digit was therefore the sole member of the other ensemble for the $\mathrm{N} 2$ condition. It is possible that for the $\mathrm{N} 2$ condition when the probed ensemble size was five, Ss recognized the digit in the test stimulus as being the digit in the nonprobed ensemble, resulting in faster RTs. No such decrease in RT occurred for the N1 condition, indicating that the Ss searched completely through the probed ensemble. These data provide some evidence that the Ss did not confine their search to only the probed ensemble.

\section{Number of Ensembles and Type of Cue}

The intercept of a function relating RT to ensemble size, when there is one ensemble in memory, is assumed to reflect the time required for stimulus encoding and response decoding. However, as Oldfield (1966) noted, when there is more than one ensemble in memory, the intercept will also reflect the time required to determine which ensemble to search. Williams (1971) supported Oldfield's suggestion for LTM by investigating the changes in the intercept constant which result from variations in the number of ensembles in memory. Williams observed that theintercept constant increased with the number of ensembles in memory. There was no interaction between the effects of the number of

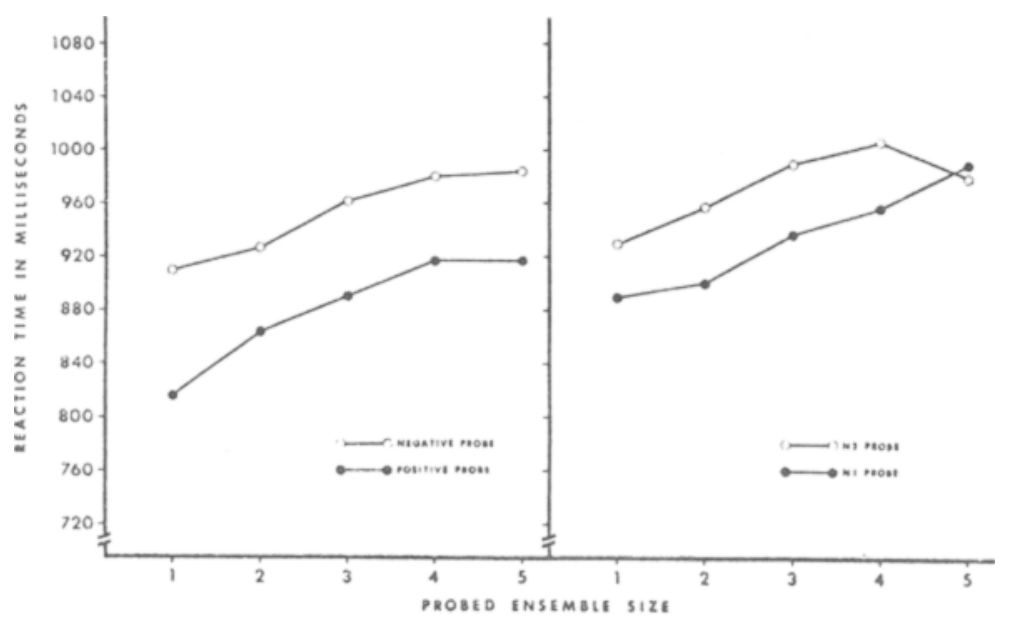

Fig. 3. Mean RT as a function of probed ensemble size for positive and negative probes (left panel) and for N1 and N2 probes (right panel) in the experimental condition. 
ensembles in memory and the memory load within ensembles, indicating that ensemble selection and ensemble scanning are indepedent processes. Also, if the increase in the intercept with increases in the number of ensembles in memory results from the additional step of serially searching for the appropriate ensemble to be scanned, then the effect of an informative cue should be to eliminate the search through the ensembles. The indication of which ensemble is to be probed should enable $S$ to be ready to search within the appropriate ensemble immediately, as when there is only one ensemble in memory. Forrin and Morin (1967), Briggs and Swanson (1969), and Kaminsky and DeRosa (1972) all found that RTs were faster with informative cues than with noninformative cues.

In order to investigate these implications further, the data for the two-ensembles and three-ensembles conditions and the informative cue and noninformative cue conditions were examined separately, as shown in Fig. 4. Reaction times for the noninformative cue condition were much slower than RTs for the informative condition, $F(1,9)=150.64, p<.001$. For the noninformative cue condition, RTs for the three-ensembles condition were consistently slower than RTs for the two-ensembles condition, $F(1,9)=39.57$, $p<.001$. The difference between the overall means for these two functions is $94 \mathrm{msec}$. For the informative cue condition, it can be seen that the difference in vertical displacement between the two functions is virtually eliminated. The difference between the mean RTs for the three-ensembles function and the two-ensembles function is only $8 \mathrm{msec}$. This difference is, however, reliable, $F(1,9)=5.28, p<.05$. Although the difference between the means is significant in a statistical sense, an 8 -msec difference is quite small relative to the difference between the functions for the noninformative cue condition.

It can also be seen in Fig. 4 that the relation between RT and probed ensemble size is different for the noninformative and informative cue conditions. In the informative condition the data support a serial search within the probed ensemble, in that RT increases with probed ensemble size for both the two-ensembles condition and the three-ensembles condition, $F(4,36)=$ $55.61, p<.001$ and $F(3,27)=50.90, p<.001$. For the two-ensembles condition only the linear component of the ensemble size variable was significant, $F(1,9)=$ $70.77, \mathrm{p}<.001$. For the three-ensembles condition both the linear and quadratic components were significant, $F(1,9)=63.80, p<.001$ and $F(1,9)=10.87, p<.01$, respectively. For the noninformative cue, two-ensembles condition, probed ensemble size was a significant variable, $F(4,36)=7.57, p<.001$. Both the linear and quadratic components were significant, $F(1,9)=7.01$, $p<.05$ and $F(1,9)=14.47, p<.005$, respectively. For the noninformative cue, three-ensembles condition, probed ensemble size was again a significant variable, $\mathrm{F}(3,27)=3.06, \mathrm{p}<.05$, but only the quadratic

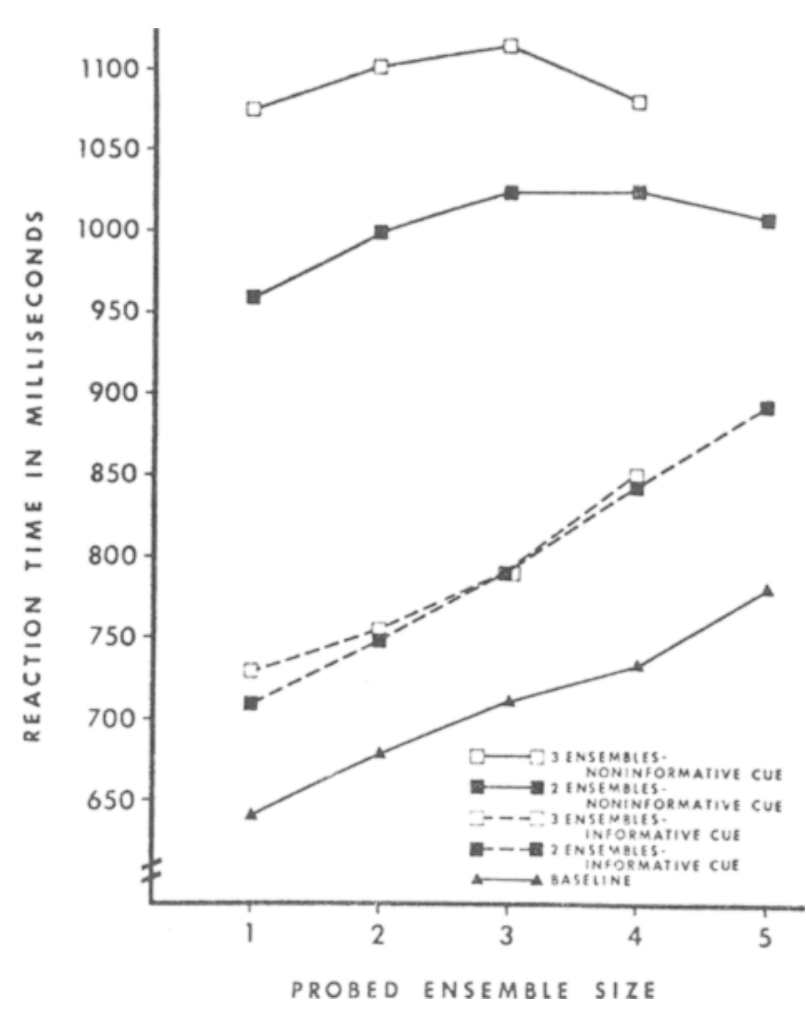

Fig. 4. Mean RT as a function of probed ensemble size in the two-ensembles and three-ensembles conditions with informative and noninformative cues.

component was significant, $\mathrm{F}(1,9)=19.06, \mathrm{p}<.005$. The slopes for the three-ensembles function and the two-ensembles function are only $3 \mathrm{msec}$ and $12 \mathrm{msec}$ per unit increase in ensemble size, respectively, compared to slopes of $40 \mathrm{msec}$ and $46 \mathrm{msec}$ per unit increase in ensemble size for corresponding functions in the informative cue condition. It appears that the search strategy used when there is no prior information as to which ensemble is to be probed is quite different from the strategy used when there is no uncertainty. When there is no informative cue, the Ss may have been performing a nondirected search through all the digits in the positive set, and then if a match was made, search was initiated through the ensemble-defining colors. Alternatively, the Ss may have been using another type of strategy entirely, or some combination of strategies. The exact nature of this search cannot be determined, but it does not appear to be as simple as that proposed by Oldfield (1966) and Williams (1971).

Another interesting finding in Fig. 4 is that RTs for the informative cue condition were still consistently slower than RTs for the baseline condition, $F(1,9)=$ $50.12, \mathrm{p}<.001$. The intercept constant for the informative cue function is $59 \mathrm{msec}$ greater than the intercept constant for the baseline function. Some of this difference may be attributed to practice effects, since the baseline condition was always run last. It appears that when there is more than one ensemble in 
memory, an additional step is required before the comparison stage can be performed, even when there is no uncertainty as to which ensemble will be probed.

\section{Grouped vs Nongrouped Presentation}

It was thought that grouping might have a facilitative effect on S's ability to organize information in STM resulting in faster RTs than for the nongrouped condition. The main effect of grouping was not found to be significant, $F(1,9)=4.68, p>.05$, nor was its interaction with any other variable significant. Therefore, this variable was not investigated further.

In summary, the present study provides evidence that Ss can utilize organization present in a series to aid retrieval from STM. However, unlike some recent studies of STM and LTM (Kaminsky \& DeRosa, 1972; Williams, 1971 ), the process is more complex when the mode of organization is independent of the items themselves and the organization is not highly overlearned. Ss do not appear to always perform a two-stage search process in which ensemble selection precedes search within the ensembles. Instead, it appears that Ss modify their search and scan not only the probed ensemble, but also, on some trials, the nonprobed ensemble. The type of search strategy used depends on whether an informative or noninformative cue precedes the test item.

\section{REFERENCES}

Briggs, G. E., \& Swanson, J. M. Retrieval time as a function of memory ensemble size. Quarterly Journal of Experimental Psychology, 1969, 21, 189-191.

Forrin, B, \& Morin, R. E. Effects of context on reaction time to optimally coded signals. Acta Psychologica, 1967, 27, 188-196.

Kaminsky, T., \& DeRosa, D. The influence of retrieval cues and set organization on short-term recognition memory. Journal of Experimental Psychology, 1972, 96, 449-454.

Milles, K. P. The effect of a basis for stimulus classification on recognition reaction time. Unpublished master's thesis, Kent State University, 1969.

Naus, M. J., Glucksberg, S., \& Ornstein, P. A. Taxonomic word categories and memory search. Cognitive Psychology, 1972, 3, 643-654.

Oldfield, B. C. Things, words, and the brain. Quarterly Journal of Experimental Psychology, 1966, 18, 340-353.

Posner, M. I. Short-term memory systems in human information processing. Acta Psychologica, 1967, 27, 267-284.

Sternberg, $S$. High speed scanning in human memory. Science, $1966,153,652-654$.

Sternberg, S. Memory-scanning: Mental processes revealed by reaction-time experiments. American Scientist, 1969, 57, $421-457$.

Williams, J. D. Memory ensemble-selection in human information processing. Journal of Experimental Psychology, $1971,88,231-238$.

(Received for publication August 10, 1973; accepted August 27, 1973.) 